\title{
Humour noir dans la pratique médicale, acceptable? A traiter avec discernement
}

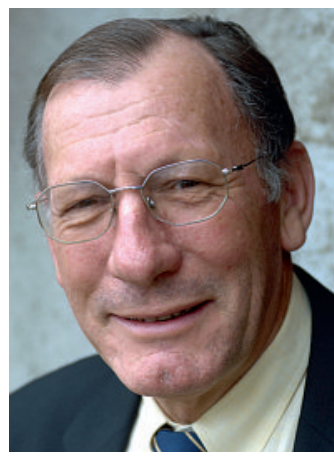

Jean Martin
«L'humour, c'est quand la vérité nous est dite plus vite et plus directement que ce à quoi nous sommes habitués» (George Saunders). Que penser du fait qu'il arrive aux médecins de faire de l'humour noir à propos des problèmes de leurs patients.? N.B.: on ne parle ici ni de plaisanteries salaces de salle de garde ni de remarques péjoratives qui seraient faites sur les patients «difficiles», mais bien d'humour (pas pensé comme insultant) en rapport avec l'état du malade ou les aléas de la prise en charge; de ce qui peut se dire entre professionnels hors de la présence du malade mais aussi avec lui, en voulant sourire d'une situation sérieuse.

Katie Watson, éthicienne et à temps partiel comédienne, qui enseigne à la Northwestern University près de Chicago, publie une étude substantielle à ce sujet [1]. D'abord, une analyse de la relation entre pouvoir et humour est nécessaire. On peut penser qu'il est permis de rire de personnes ou groupes plus importants que nous mais que l'inverse ne l'est pas. C'est ce qui permet de se ficher des professeurs durant une fête de fin d'études. Aussi rire du patron (en coulisses) peut être une manière d'éviter d'entrer en conflit avec lui.

La plaisanterie représente ainsi souvent une sorte de rébellion contre une autorité, oppressante le cas échéant, et il y a peu d'autorités qui le sont plus que la mort, la maladie, le traumatisme - dans les camps de concentration, il arrivait que les prisonniers rient de leurs inhumaines conditions. Un rôle de l'humour est de mettre le doigt sur et d' «intégrer» des absurdités douloureuses, ou pour les moins des incongruités. Un effort de rester sensé dans un monde insensé - ce qui arrive aussi dans des situations cliniques!

L'argument peut être avancé que l'activité de médecin est si lourde que tout est alors permis dans les coulisses, notamment dans l'optique de faire face (coping), mais cet argument doit être évalué attentivement. Il ne saurait tout excuser mais a sa part de pertinence. L'humour noir est une manière de gérer, de digérer des situations dramatiques secouantes, aidant à se rétablir en vue de poursuivre sa tâche avec d'autres patients. Watson rapporte le cas de jeunes médecins en service d'urgence ayant commandé des pizzas à l'extérieur, dont le livreur est agressé par arme à feu sur la rue en les apportant... Après avoir vainement cherché à le sauver et dans le stress du moment, l'un d'entre eux demande: «A votre avis, quel pourboire aurions-nous dû lui donner?», suscitant un fou rire. Discutable. Mais (Watson), «si je suis le patient de l'ambulance suivante, arrivant après mon accident, mon infarctus, un viol, je serai heureux qu'il ait fait ce gros gag. Parce que ces internes en avaient besoin pour continuer à travailler [...] L'humour noir peut être une manifestation de respect pour le travail qui est au-devant de soi.» C'est une façon de prendre du recul.

Watson cite une psychiatre senior qui dit plaisanter parfois à propos de patients mais considère que les plus jeunes n'ont pas encore gagné le droit de le faire... Remarque discriminatoire mais qui peut aussi paraître de bon sens; l'expérience (qui ne s'apprend pas dans les livres!) est ce qui permet de mieux jauger une situation, d'évaluer ce qu'il est possible de faire ou pas. Mais il y a d'autres aspects: les jeunes médecins, ou les étudiants en salle de dissection, utilisent plus souvent que leurs confrères expérimentés l'humour noir comme moyen de faire baisser la tension (mais, N.B., la situation peut être inverse chez certains, s'agissant des remarques méprisantes à propos des malades - qui ne sont pas le sujet du présent papier). Noter ici que, en début de carrière, les jeunes médecins et soignants sont souvent plus directement au contact des soucis, tristesses, frustrations voire colères des patients et de leurs familles, donc moralement plus sollicités.

On peut parfois dire qu'on plaisante d'une situation mais pas vraiment d'un malade; pas forcément une bonne excuse - et comment faire la différence? Alors, humour noir dans la pratique des soins? Possible oui, avec discernement. On ne doit toutefois pas exclure un «humour thérapeutique» entre soignants et soignés: façon de détendre la situation tout en signifiant qu'on se bat ensemble, et reconnaissance de notre commune et bien imparfaite condition humaine. Watson remarque que «la médecine est une drôle de profession, dans laquelle on demande à des gens ordinaires d'agir comme si faeces ou vomi ne sentent rien, comme si des corps altérés ne le sont pas et comme si la mort n'était pas effrayante»...

La question reste, quand le fait de plaisanter est-il une forme d'abus? Pour l'auteure s'il n'y pas lieu d'excommunier l'humour noir en médecine, nous sommes en terrain glissant ( $W$ We are on thin ice»). Les professionnels de soins ont le constant devoir de faire preuve de suffisamment d'ouverture, de tact, de pondération, y compris et surtout lorsqu'ils entendent plaisanter. Parce que, dit Nell Kirst [2] dans un texte de la même revue, c'est le "prix que nous avons à payer pour l'autorisation que les patients nous donnent d'entrer dans les espaces sacrés de leur vies».

Jean Martin, membre de la Commission nationale d'éthique et de la rédaction du BMS 